Ocular Oncology

and Pathology
Ocul Oncol Pathol 2016;2:178-180

DOI: $10.1159 / 000443650$
Received: October 1, 2015

Accepted after revision: December 8, 2015

Published online: January 27, 2016

\title{
Optic Nerve Assessment Using 7-Tesla Magnetic Resonance Imaging
}

\author{
Arun D. Singh ${ }^{a}$ Sean M. Platt ${ }^{a}$ Lisa Lystad ${ }^{a}$ Mark Lowe $^{b}$ Sehong Oh ${ }^{b}$ \\ Stephen E. Jones ${ }^{\mathrm{b}}$ Yahya Alzahrani ${ }^{\mathrm{a}}$ Thomas Plesec ${ }^{\mathrm{c}}$ \\ ${ }^{a}$ Department of Ophthalmic Oncology, Cole Eye Institute, ${ }^{b}$ Imaging Institute, and ${ }^{c}$ Pathology Institute, \\ Cleveland Clinic, Cleveland, Ohio, USA
}

\section{Key Words}

7-tesla magnetic resonance imaging $\cdot$ Choroidal malignant melanoma. Optic nerve

\begin{abstract}
Purpose: The purpose of this study was to correlate highresolution magnetic resonance imaging (MRI) and histologic findings in a case of juxtapapillary choroidal melanoma with clinical evidence of optic nerve invasion. Methods: With institutional review board approval, an enucleated globe with choroidal melanoma and optic nerve invasion was imaged using a 7-tesla MRI followed by histopathologic evaluation. Results: Optical coherence tomography, B-scan ultrasonography, and 1.5-tesla MRI of the orbit (1-mm sections) could not detect optic disc invasion. Ex vivo, 7-tesla MRI detected optic nerve invasion, which correlated with histopathologic features. Conclusions: Our case demonstrates the potential to document the existence of optic nerve invasion in the presence of an intraocular tumor, a feature that has a major bearing on decision making, particularly for consideration of enucleation.

(c) 2016 S. Karger AG, Basel
\end{abstract}

\section{Introduction}

High-resolution magnetic resonance imaging (MRI) is emerging as a tool for assessing ocular structures $[1,2]$. The eye is an ideal organ to be examined by MRI given the difference in water content of ocular structures [2]. Using specialized head coils for imaging, the qualities of ocular and orbital images produced with 1.5 and $3.0 \mathrm{~T}$ have improved, but they do not provide the detailed imaging afforded by 7-tesla imaging [3]. Herein, we present a case of juxtapapillary choroidal melanoma with clinical evidence of optic nerve invasion. Following enucleation, ex vivo 7-tesla MRI findings and histopathology were correlated.

\section{Case Description}

A 74-year-old male was referred for reduced vision in the left eye. Visual acuity with correction was 20/20 OD and 20/50 OS. Fundus examination revealed a macular choroidal melanoma (8.0 $\times 7.5 \times 2.5 \mathrm{~mm}$ ) with optic disc extension from 3 to 5 o'clock (fig. 1a). Spectral-domain optical coherence tomography, B-scan ultrasonography, and 1.5-tesla MRI of the orbit (1-mm sections) could not detect optic disc invasion (fig. 1b). The eye was enucleated without complications.

\section{KARGER}

E-Mail karger@karger.com www.karger.com/oop
(C) 2016 S. Karger AG, Base

2296-4681/16/0023-0178\$39.50/0
Arun D. Singh, MD

Department of Ophthalmic Oncology, Cole Eye Institute

Cleveland Clinic, 9500 Euclid Ave, I32

Cleveland, $\mathrm{OH} 44118$ (USA)

E-Mail singha@ccf.org 
Fig. 1. In vivo imaging. Color fundus photo of juxtapapillary choroidal melanoma (8.0 $\times 7.5 \times 2.5 \mathrm{~mm}$ ) with optic nerve extension from 3 to 5 o'clock (a). 1.5-tesla MRI demonstrating the presence of a tumor in proximity to the optic disc without any evidence of optic nerve invasion (b).
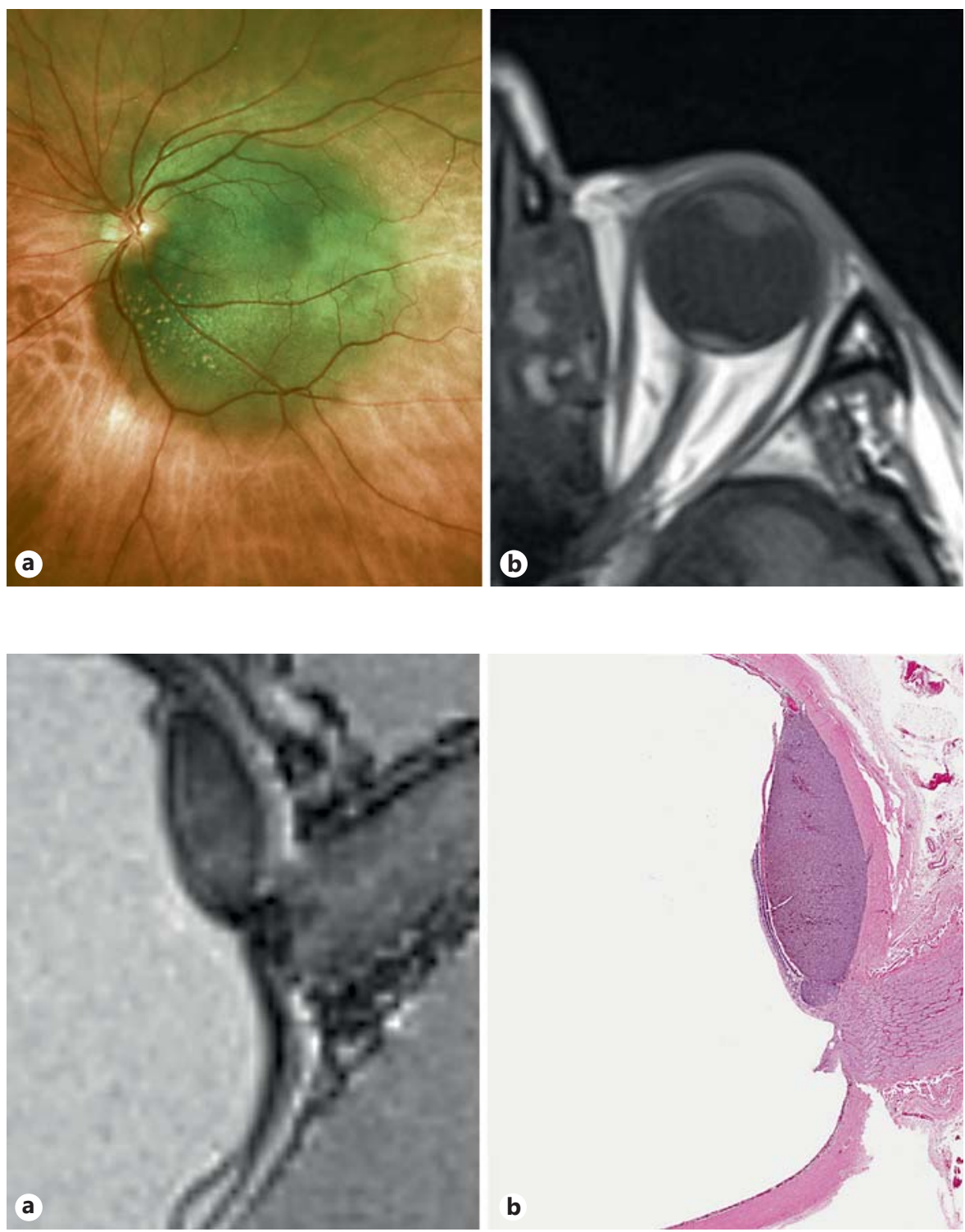

Fig. 2. Ex vivo imaging. 7-tesla MRI of the same globe after enucleation. Prelaminar optic nerve extension is detectable (a). Photomicrograph of a pupil-optic nerve section with comparable axial orientation. Choroidal melanoma is seen impinging on the prelaminar optic nerve $(\mathbf{b}$; HE, original magnification $\times 40)$.

\section{Discussion}

The globe was fixed in $10 \%$ buffered formalin. Ex vivo MRI was performed using a Siemens 7-tesla SC72 scanner with an AG 830AS magnet, used with a Nova Medical single-channel transmit and a 32-channel receiver head coil under an institutional review boardapproved protocol. The acquisition parameters were a MP2RAGE sequence with a repetition time $=6,000 \mathrm{~ms}$, an echo time $=4.42 \mathrm{~ms}$, inversion time $\mathrm{TI}_{1} / \mathrm{TI}_{2}=700 / 2,600 \mathrm{~ms}$, and flip angle $\mathrm{FA}_{1} / \mathrm{FA}_{2}=4 / 5^{\circ}$. Total acquisition time was $8 \mathrm{~h}$ ( $19.2 \mathrm{~min} \times 25$ repetitions). Voxel size was $0.25 \mathrm{~mm} \times 0.25 \mathrm{~mm}$, with a $0.25-\mathrm{mm}$ slice thickness (fig. $2 \mathrm{a}$ ).

\section{Histopathology}

A pupil-optic nerve section was obtained. Juxtapapillary mixed-cell-type melanoma was identified. Extension into the prelaminar optic nerve was observed (fig. 2b).

Optic Nerve Assessment Using MRI
Uveal melanoma extension into the optic nerve is uncommon, and its presence is seen in $0.6-5 \%$ of enucleated eyes [4]. Current techniques to image the optic disc such as optical coherence tomography, ultrasound, and computed tomography scan do not offer reliable assessment. In a review of 60 cases of retinoblastoma, a tumor with a propensity to invade the optic nerve, MRI (1.5 and 3.0 T) findings correlated with histopathologic evidence only in $44 \%$ of the cases [5].

Ultrahigh-field MRI such as $7 \mathrm{~T}$ affords a submillimeter spatial resolution and improved signal-to-noise ratios 
when compared to lower-field strength MRI commonly available at most institutions [6]. Recent attempts to increase the resolution for in vivo imaging have included the use of 7-tesla MRI with a custom-designed coil array that are expensive and are sensitive to motion artifacts [3]. Optoacoustic triggering schemes and normalization of imaging parameters such as pulse repetition time, echo time, bandwidth, and reconstruction matrix can overcome such limitations and provide high-resolution images of the optic nerve from the disc to the orbital apex $[2,6]$.

Even though 7-tesla MRI was done ex vivo, the purpose of our investigation was to assess the clinical, radiologic, and histopathologic correlations in a pathologic state. In vivo imaging with 1.5 -tesla MRI did not detect any optic nerve invasion in contrast to 7-tesla MRI, which correlated with histopathologic features. Extended scanning times in our case were related to using large-bore gradient and radiofrequency coils and the development of globe imaging protocols, as this was the first 7-tesla MRI performed on a globe at our institution. Beenakker et al. [3] and Lindner et al. [6] have previously demonstrated submillimeter resolution of the globe with 3- and 2.5-min acquisition times, respectively, using custom-designed dedicated eye coils. Although 7-tesla MRI of the optic nerve has been described previously [6], to our knowledge, our report is the first attempt at histopathologic correlation. Our case demonstrates the potential to document the existence of optic nerve invasion in the presence of an intraocular tumor, a feature that has a major bearing on decision making particularly for consideration of enucleation.

Currently, 7-tesla MRI is investigational and not FDA approved for clinical use. The study of a large number of cases will be required to fully assess the clinical utility over existing 1.5- or 3-tesla MRI capabilities.

\section{Statement of Ethics}

The authors have no ethical conflicts to disclose.

\section{References}

1 Townsend KA, Wollstein G, Schuman JS Clinical application of MRI in ophthalmology. NMR Biomed 2008;21:997-1002.

2 Bert RJ, Patz S, Ossiani M, et al: High-resolution MR imaging of the human eye 2005. Acad Radiol 2006;13:368-378.

3 Beenakker JW, van Rijn GA, Luyten GP, Webb AG: High-resolution MRI of uveal melanoma using a microcoil phased array at $7 \mathrm{~T}$. NMR Biomed 2013;26:1864-1869.

4 Lindegaard J, Isager P, Prause JU, Heegaard S: Optic nerve invasion of uveal melanoma. APMIS 2007;115:1-16.
5 Wilson MW, Rodriguez-Galindo C, Billups C, Haik BG, Laningham F, Patay Z: Lack of correlation between the histologic and magnetic resonance imaging results of optic nerve involvement in eyes primarily enucleated for retinoblastoma. Ophthalmology 2009;116: 1558-1563.

6 Lindner T, Langner S, Graessl A, et al: High spatial resolution in vivo magnetic resonance imaging of the human eye, orbit, nervus opticus and optic nerve sheath at 7.0 Tesla. Exp Eye Res 2014;125:89-94.
Singh/Platt/Lystad/Lowe/Oh/Jones/ Alzahrani/Plesec 